\title{
1 Suppressors of YpsA-mediated cell division inhibition in Bacillus subtilis
}

2 Robert S. Brzozowski¹, Brooke R. Tomlinson¹, Michael D. Sacco², Judy J. Chen ${ }^{1}$, Anika N. Ali , Yu Chen²,

3 Lindsey N. Shaw ${ }^{1}$, Prahathees J. Eswara ${ }^{1, *}$

4 1Department of Cell Biology, Microbiology and Molecular Biology, University of South Florida, Tampa, FL

533620, USA

62 Department of Molecular Medicine, University of South Florida, Tampa, FL 33612, USA

$7 \quad$ *To whom correspondence should be addressed. Email: eswara@usf.edu

8 Keywords: FtsZ; GpsB; filamentation; SLOG; YfhS

9 Running title: Suppressors of YpsA-induced filamentation in B. subtilis

\section{GRAPHICAL ABSTRACT}

Isolation of spontaneous suppressors

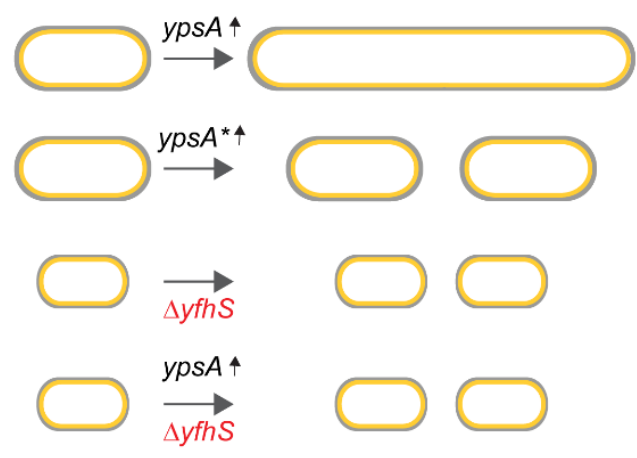

$\wedge$ Loss of $y$ fhS abolishes YpsA-mediated cell division inhibition.

$\wedge$ YfhS is a previously unknown cell size regulator.

\section{ABBREVIATED SUMMARY}

14 In Bacillus subtilis, we discovered that increased expression of ypsA results in cell

15 division inhibition and impairment of colony formation on solid medium. Colonies that do

16 arise possess compensatory suppressor mutations. Analysis of one such suppressor

17 mutation led us to a protein of unknown function, YfhS, which appears to play a role in 18 regulating cell length and cell width. 


\section{SUMMARY}

20 Although many bacterial cell division factors have been uncovered over the years,

21 evidence from recent studies points to the existence of yet to be discovered factors

22 involved in cell division regulation. Thus, it is important to identify factors and conditions

23 that regulate cell division to obtain a better understanding of this fundamental biological

24 process. We recently reported that in the Gram-positive organisms Bacillus subtilis and

25 Staphylococcus aureus, increased production of YpsA resulted in cell division inhibition.

26 In this study, we isolated spontaneous suppressor mutations to uncover critical residues

27 of YpsA and the pathways through which YpsA may exert its function. Using this

28 technique, we were able to isolate four unique intragenic suppressor mutations in yps $A$

29 (E55D, P79L, R111P, G132E) that rendered the mutated YpsA non-toxic upon

30 overproduction. We also isolated an extragenic suppressor mutation in $y f h S$, a gene

31 that encodes a protein of unknown function. Subsequent analysis confirmed that cells

32 lacking $y$ fhS were unable to undergo filamentation in response to YpsA overproduction.

33 We also serendipitously discovered that YfhS may play a role in cell size regulation.

\section{INTRODUCTION}

36 Bacterial cell division is an essential process orchestrated by a multitude of cell division

37 proteins (Haeusser \& Margolin, 2016). During growth an essential cell division protein

38 FtsZ, forms a ring-like structure and marks the site of division. There it serves in the

39 recruitment of additional divisome proteins and commences septation (Du \&

40 Lutkenhaus, 2017, Errington \& Wu, 2017). Although known FtsZ regulatory systems,

41 such as the Min system and nucleoid occlusion, have been well characterized (Eswara 
42 \& Ramamurthi, 2017), recent studies have determined that correct cell division site

43 selection can occur independent of these mechanisms in both Bacillus subtilis and

44 Escherichia coli (Rodrigues \& Harry, 2012, Bailey et al., 2014). These findings highlight

45 the need to investigate and discover other factors involved in regulating cell division in

46 bacteria. In our lab, we have identified a potential cell division regulator in $B$. subtilis and

47 Staphylococcus aureus, YpsA (Brzozowski et al., 2019a).

49 YpsA is conserved in the Firmicutes phylum of Gram-positive bacteria and appears to 50 be in a syntenous relationship with a known cell division protein, GpsB (Brzozowski et

51 al., 2019a). The crystal structure of $B$. subtilis YpsA was solved by a structural

52 genomics group in 2006 [PDB ID: 2NX2; (Ramagopal et al., 2006)]. Based on the

53 structural features, YpsA was placed as the founding member of the "YpsA proper"

54 subclade within the SLOG (MF/DprA/LOG) protein superfamily (Burroughs et al.,

55 2015), yet the precise function of YpsA remains to be elucidated. The structure of YpsA

56 resembles that of DprA (RMSD with DprA of Helicobacter pylori is $2.79 \AA$, PDB ID:

57 4LJR), another member of the SLOG superfamily, which is a single-stranded DNA-

58 binding protein involved in DNA recombination (Yadav et al., 2014, Wang et al., 2014).

59 Previously we had found that YpsA provides oxidative stress protection in B. subtilis and

60 that overproduction of YpsA results in cell division inhibition, through FtsZ

61 mislocalization, in a growth rate dependent manner (Brzozowski et al., 2019a).

62 Additionally, using site-directed mutagenesis we identified multiple amino acid residues

63 that are potentially important for the structure and/or function of YpsA, including

64 residues located in the conserved substrate binding pocket made up of glycine and 
glutamate residues predicted by Burroughs et al., 2015 (Burroughs et al., 2015). In addition, we have shown that the function of YpsA in cell division is also conserved in

67 the Gram-positive pathogen, S. aureus (Brzozowski et al., 2019a). In this study we utilized a classic spontaneous suppressor isolation technique for further

70 identification of critical amino acid residues that are important for YpsA structure and/or

71 function, and for the elucidation of the molecular mechanism through which YpsA acts.

72 By screening for suppressor mutations of a lethal YpsA overproduction phenotype, we

73 were able to isolate and characterize four unique intragenic suppressor mutations

74 (E55D, P79L, R111P, G132E). Each of these mutations was found to prevent lethality

75 and related cell division inhibition. In addition, we also identified an extragenic

76 suppressor mutation that introduced a premature stop codon in the $y$ fhS gene, which

77 codes for a protein of unknown function. Upon subsequent analysis, we verified that in

78 cells lacking $y$ fhS, YpsA-dependent cell division inhibition is abolished. Here, we

79 speculate the possible ways by which YfhS and YpsA-mediated cell division phenotypes

80 could be linked. Interestingly, during the course of our experiments, we discovered that

81 YfhS may play a role in cell size regulation, as $y$ fhS null cells are significantly smaller in

82 cell width and length when compared to the wild type control.

\section{RESULTS}

85 Overexpression of $y p s A$ results in a growth defect on solid medium

86 We have previously shown that overproduction of either YpsA or YpsA-GFP results in

87 severe filamentation in B. subtilis [(Brzozowski et al., 2019a); Fig. 1A)], a phenotype 
88 that is characteristic of cell division inhibition in this organism. To test whether

89 filamentous growth in the presence of inducer results in a distinguishable phenotype on

90 solid medium, we conducted a spot assay. Briefly, serial dilutions of exponentially

91 growing wild type (WT) cells, and cells containing an IPTG-inducible copy of either ypsA

92 or ypsA-gfp were spotted on solid growth medium with or without inducer. In the

93 absence of inducer all strains of all tested dilutions grew similar to the WT control (Fig.

94 1B; see left panel). In the presence of inducer, we observed a significant growth defect

95 associated with both YpsA and YpsA-GFP overproduction, suggesting that cell division

96 inhibition caused by ypsA or ypsA-gfp overexpression was lethal (Fig. 1B; see right

97 panel). Interestingly, YpsA-GFP overproduction resulted in a more severe growth

98 phenotype compared to untagged YpsA. To test whether this difference is due to

99 increased accumulation of YpsA-GFP in the cells, we tagged both YpsA and YpsA-GFP

100 with a FLAG tag at their C-terminus and conducted an anti-FLAG western blot analysis.

101 Overproduction of YpsA-FLAG and YpsA-GFP-FLAG resulted in filamentation and a

102 growth defect on solid medium that was of similar extent when compared to their non-

103 FLAG tagged counterparts (Figs. S1A and S1B). The ratio of YpsA-GFP-FLAG and

104 SigA (internal loading control) was similar to that of YpsA-FLAG and SigA (0.68 and

1050.80 respectively; Fig. S1C), suggesting that the increased lethality of the GFP-tagged

106 version is not due to any changes in accumulation. Next, we utilized the severe lethality

107 elicited by YpsA-GFP overproduction as a tool to isolate spontaneous suppressors.

108

109 Isolation of spontaneous suppressor mutations 
110 The YpsA-GFP overproducing strain was streaked out for single colony isolation on

111 multiple inducer containing plates and the plates were incubated overnight as described

112 in the methods section. Only a few colonies formed per plate, presumably due to

113 spontaneous suppressor mutations, which allow for normal growth despite the presence

114 of inducer. After multiple iterations of suppressor isolation, likely mutations were

115 subsequently determined to be either intragenic (within inducible ypsA-gfp, henceforth

116 noted as yps $A^{*}$-gfp for simplicity) or extragenic (elsewhere on the chromosome), and

117 their chromosomes were sequenced to identify the mutations (Fig. S2). Using this

118 approach, we were able to isolate four unique intragenic suppressor mutations: G132E,

119 P79L, R111P, and E55D (listed in the order of isolation). Immunoblotting indicated that

120 these mutant versions of YpsA were stably produced (Fig. 2L). Next, fluorescence

121 microscopy was used to determine whether these mutations were able to rescue the

122 lethal filamentous phenotype observed when unmutated YpsA-GFP was overproduced.

123 This was carried out on exponentially growing cells of the ypsA-gfp overexpression

124 strain and all yps $A^{*}$-gfp intragenic suppressor strains in the absence or presence of

125 inducer. In the absence of inducer all strains exhibited similar cell lengths [YpsA-GFP:

$1263.23 \pm 0.74 \mu \mathrm{m}$ (Fig. 2A); G132E: $3.49 \pm 0.93 \mu \mathrm{m}$ (Fig. 2C); P79L: $3.24 \pm 0.81 \mu \mathrm{m}$ (Fig.

127 2E); R111P: $3.16 \pm 0.76 \mu \mathrm{m}$ (Fig. 2G); E55D: $3.23 \pm 0.83 \mu \mathrm{m}$ (Fig. 2I) | $\mathrm{n}=100$ for all cell

128 length measurements]. Upon the addition of inducer, we found that cells overexpressing

$129 y p s A^{*}$-gfp did not exhibit filamentation, unlike the ypsA-gfp control [YpsA-GFP: $8.93 \pm$

$1305.67 \mu \mathrm{m}$ (Fig. 2B); G132E: $2.99 \pm 0.88 \mu \mathrm{m}$ (Fig. 2D); P79L: $3.29 \pm 0.81 \mu \mathrm{m}$ (Fig. 2F);

131 R111P: $3.06 \pm 0.98 \mu \mathrm{m}$ (Fig. 2H); E55D: $3.10 \pm 0.83 \mu \mathrm{m}$ (Fig. 2J)], indicating that the

132 intragenic suppressors were unable to elicit filamentation upon overproduction. We also 
133 noted that G132E, P79L, and R111P suppressors displayed impaired foci formation in

134 comparison to the ypsA-gfp control (Figs. 2B, 2D, 2F, and 2H). However, foci formation

135 in E55D suppressor was not impaired (Fig. 2J).

137 Each of the intragenic suppressors were subjected to a spot assay to test whether these

138 point mutations were able to grow normally in contrast to the ypsA-gfp overexpression

139 strain that displayed a lethal phenotype on solid medium in the presence of inducer. In

140 the absence of inducer all strains grew similar to the WT control (Fig. 2K; see top

141 panel). When grown in the presence of inducer, overexpression of ypsA-gfp resulted in

142 a severe growth defect (Fig. 2K; see bottom panel). However, growth was similar to WT

143 in all intragenic suppressor strains when grown in the presence of inducer (Fig. 2K; see

144 bottom panel). Given that these mutants were unable to cause filamentation, it appears

145 that the lethality is directly linked to the ability of YpsA to elicit filamentation. Collectively,

146 these data indicate that the residues E55, P79, R111, and G132 are critical for the

147 function of YpsA, especially in regard to cell division inhibition.

Structural analysis of the intragenic suppressor mutations

150 Three of the four YpsA mutants have residues that are buried in the core: E55D,

151 G132E, and P79L (Fig. 3A). When large mutations occur in this environment, misfolding

152 and loss of function is often the consequence (Baruah \& Biswas, 2014). Amongst these

153 mutants, P79 is significant because it, and its adjacent residue F80, are strictly

154 conserved amongst the YpsA clade of Firmicutes (Burroughs et al., 2015, Brzozowski et

155 al., 2019a). The crystal structure of $B$. subtilis YpsA (PDB ID: 2NX2) reveals P79 and 
156 F80 also line the possible DNA binding groove of YpsA (Ramagopal et al., 2006). The

157 positioning of an aromatic side chain here suggests it may facilitate DNA binding by

158 forming stacking interactions with nucleobases (Baker \& Grant, 2007). The P79L

159 mutation not only creates severe clashes with surrounding residues, but likely perturbs

160 the positioning of F80, and therefore may directly impair DNA or nucleotide binding (Fig.

161 3B). Likewise, the E55D mutant affects a second, highly conserved segment of the

162 putative DNA binding domain, the GxE motif. In YpsA, E55 is highly coordinated by five

163 potential hydrogen bonds with the side chain and backbone of S49, the T7 sidechain,

164 and the backbone amide of Q51 (Fig. 3C). Considering the size and physicochemical

165 properties of their sidechains, one would expect an E $\rightarrow$ D mutation to have a non-

166 deleterious effect on YpsA. However, in this instance, shortening the sidechain of E55

167 by a methylene results in the weakening or total loss of these hydrogen bonds and likely

168 destabilizes the possible DNA binding groove. In addition, the aliphatic part of the E55

169 side chain forms extensive van der Waals interactions with nearby residues such as

170 Q51 and others, and the carboxylate group of an aspartate residue at this position

171 would also clash with these surrounding residues. The third core mutation, G132E, is

172 located at the beginning of a $\beta$-strand and is surrounded by multiple bulky and

173 hydrophobic residues including Y164, P162, L1, L4, and F38 (Fig. 3D). Conversion from

174 glycine to any other residue besides alanine results in clashes that will affect the

175 secondary structural elements from which the surrounding residues originate. Indeed,

176 every possible G132E rotamer produces significant clashes, with interatomic distances

177 less than $2.2 \AA$. 
179 The R111P mutation is the only intragenic suppressor mutation that involves a solvent exposed residue. Here R111 normally forms a salt bridge with the neighboring E114

181 (Fig. 3E). The conversion from a positively charged side chain to a nonpolar one

182 eliminates this interaction. Furthermore, the cyclic nature of the proline side chain

183 introduces a steric clash with the backbone amide nitrogen of its adjacent residue,

$184 \mathrm{H} 110$. The R111P mutation will likely force conformational changes in the protein

185 backbone and cause significant disruptions in intramolecular interactions involving

186 nearby residues. A second scenario that leads to the disruption of YpsA function in this

187 mutant involves the impairment of intermolecular interactions and macromolecular

188 recognition. It is possible that a mutation from $\mathrm{R} \rightarrow \mathrm{P}$ prevents interaction with other

189 protein partners of YpsA.

\section{Isolation and validation of an extragenic suppressor mutation in yfhS}

192 Using the same suppressor screening approach (Fig. S2), we were able to isolate and

193 validate an extragenic suppressor mutation, which is a duplication of a stretch of 10

194 nucleotides that introduces a premature stop codon in $y f h S$ (Fig. S3A). YfhS is a 74

195 amino acid containing protein of unknown function. yfhS is annotated as a sporulation

196 gene upregulated by SigE sigma factor during sporulation (Zhu \& Stulke, 2018).

197 However, there is no sporulation defect in a yfhS null strain (Yamamoto et al., 1999).

$198 y$ fhS may also be regulated by the transcription factor AbbA (Banse et al., 2008).

200 To test whether disruption of $y f h S$ restores normal cell length in cells overexpressing

201 either ypsA or ypsA-gfp, we generated a strain harboring an inducible copy of either 
$202 y p s A$ or $y p s A-g f p$ in a $y f h S$ null background. These strains were then screened with the

203 appropriate controls via a spot assay in order to observe whether or not the $y f h S$

204 deletion was able to restore normal growth on solid medium even when YpsA or YpsA-

205 GFP was overproduced. In the absence of inducer, WT cells and cells containing an

206 inducible copy of either ypsA or ypsA-gfp grew similarly (Fig. 4A). Cells lacking yfhS

207 formed small colonies in comparison to WT suggesting an intrinsic growth phenotype

208 associated with the deletion of $y$ fhS. Cells harboring an inducible copy of ypsA or ypsA-

209 gfp in a $y f h S$ null background grew similar to the $y f h S$ null control strain. When grown in

210 the presence of inducer, as shown in Fig. 1B, cells harboring an inducible copy of ypsA

211 showed a moderate growth defect while inducible ypsA-gfp strain exhibited a severe

212 growth defect (Fig. 4B). In the presence of inducer, cells harboring a yfhS knockout and

213 cells harboring a $y f h S$ knockout with an inducible copy of either ypsA or ypsA-gfp grew

214 similarly, suggesting that deletion of $y$ fhS prevents elicitation of lethal phenotypes

215 displayed by YpsA or YpsA-GFP overproducing cells (Fig. 4B). To ensure the

216 phenotype was specific to the disruption of the native copy of $y f h S$, we introduced $y f h S$

217 at an ectopic locus under an inducible promoter. In this complementation strain, the

218 presence of inducer or even leaky expression in the absence of inducer, restored WT-

219 like growth (Fig. 4A). Interestingly, the defective growth phenotype of YpsA and YpsA-

220 GFP overproducing cells was also restored in the presence of inducer in the

221 complementation strain (compare Figs. 4A and 4B).

222

223 Next, we inspected the cell morphology of all strains tested in Figs. 4A and 4B through

224 fluorescence microscopy. Cell division in cells harboring an inducible copy of either 
$225 y p s A$ or $y p s A-g f p$, but not in WT control, were inhibited upon the addition of inducer

226 (Fig. 4C-H), as discussed previously [Fig. 1A; (Brzozowski et al., 2019a)]. The

227 quantification of cell lengths are shown in Fig. 4U. The cell lengths of the WT control

228 strain in the absence and presence of inducer were similar [WT (- inducer): $2.72 \pm 0.68$

$229 \mu \mathrm{m}$ (Fig. 4C); WT (+ inducer): $2.77 \pm 0.69 \mu \mathrm{m}$ (Fig. 4D)]. On the contrary, as expected,

230 cells overproducing YpsA or YpsA-GFP exhibited filamentation in the presence of

231 inducer [YpsA (- inducer): $2.59 \pm 0.71 \mu \mathrm{m}$ (Fig 4E); YpsA (+ inducer): $6.36 \pm 2.56 \mu \mathrm{m}$

232 (Fig 4F) | YpsA-GFP (- inducer): $2.99 \pm 0.73 \mu \mathrm{m}$ (Fig. 4G); YpsA-GFP (+ inducer): 6.77

$233 \pm 3.48 \mu \mathrm{m}$ (Fig. 4H)].

235 Upon imaging the $\Delta y f h S$ cells, to our astonishment, we noticed that the average cell 236 length was smaller than WT cells [ $\Delta y$ fhS (- inducer): $1.99 \pm 0.61 \mu \mathrm{m}]$, compare Figs. 4I

237 and 4C. In addition to a smaller cell length, the average cell width of $\Delta y f h S$ cells also

238 appeared to be smaller when compared to WT [WT (- inducer): $0.78 \pm 0.06 \mu \mathrm{m}$ (Fig.

239 4C); $\Delta y f h S$ (- inducer): $0.67 \pm 0.08 \mu \mathrm{m}$ (Fig. 4I) $\mid \mathrm{n}=100]$ ]. This observation hints at the

240 possible role for YfhS in cell size regulation either directly or indirectly. Addition of

241 inducer had no effect on the average cell length of cells lacking $y f h S[\Delta y f h S(+$ inducer):

$2421.91 \pm 0.48 \mu \mathrm{m} ;$ Fig. 4J]. When cells containing an inducible copy of either yps $A$ or

$243 y p s A-g f p$ in a yfhS null background were imaged, they also exhibited smaller cell

244 lengths in the absence of inducer $[\Delta y f h S+$ YpsA (- inducer): $1.93 \pm 0.52 \mu \mathrm{m}$ (Fig. 4K);

$245 \Delta y f h S+Y p s A-G F P(-$ inducer): $2.02 \pm 0.55 \mu \mathrm{m}$ (Fig. 4M)], suggesting that that small-

246 cell phenotype is intrinsically linked to the lack of the $y f h S$ gene. Intriguingly,

247 overproduction of either YpsA or YpsA-GFP did not result in filamentation in a yfhS null 
248 background $[\Delta y f h S+$ YpsA (+ inducer): $1.97 \pm 0.56 \mu \mathrm{m}$ (Fig. 4L); $\Delta y f h S+$ YpsA-GFP $(+$

249 inducer): $2.17 \pm 0.72 \mu \mathrm{m}$ (Fig. $4 \mathrm{~N}$ ), indicating that YpsA-mediated cell division inhibition

250 is dependent on YfhS. The ratio of YpsA-GFP-FLAG and SigA in the WT background

251 and $y$ fhS null background were similar ( 0.68 and 0.56 respectively; Fig. S1C),

252 suggesting that the elimination of cell division inhibition is not due to defective

253 accumulation of YpsA-GFP. We did observe a 2-fold increase in the ratio of YpsA-FLAG

254 and SigA between WT and $\Delta y f h S$ background ( 0.80 and 1.59 respectively; Fig. S1C).

255 However, this can be attributed to lower levels of SigA that we have seen reproducibly

256 in this strain background, when the optical density is standardized between the strains

257 tested. Thus, we conclude that the abolition of cell division inhibition in yfhS null strain is

258 not due to defective accumulation of YpsA or YpsA-GFP.

259

260 We further confirmed that the $y$ fhS deletion phenotype is linked specifically to $y f h S$, and

261 not due to any kind of polar effect, by using the complementation strain described

262 earlier. Fluorescence microscopy revealed that the characteristic small-cell phenotype

263 of $\Delta y$ fhS was no longer observed, even in the absence of inducer, likely due to the leaky

264 expression of $y h f S$ in the complementation strain $[\Delta y f h S+y f h S(-$ inducer): $2.23 \pm 0.58$

$265 \mu \mathrm{m}$ (Fig. 40)]. When the expression of ectopic $y$ fhS was induced by the addition of

266 inducer, the average cell length resembled that of the WT control $[\Delta y f h S+y f h S(+$

267 inducer): $2.47 \pm 0.65 \mu \mathrm{m}$ ], compare Figs. 4P and 4D. As expected, cells carrying an

268 IPTG-inducible copy of ypsA or ypsA-gfp in the complementation strain background, in

269 the absence of inducer, appeared similar to the WT control $[\Delta y f h S+y f h S+$ YpsA $(-$

270 inducer): $2.31 \pm 0.76 \mu \mathrm{m}$ (Fig. 4Q); $\Delta y f h S+y f h S+$ YpsA-GFP (- inducer): $2.44 \pm 0.80$ 
$271 \mu \mathrm{m}$ (Fig. 4S)]. However, in the presence of inducer filamentation was restored in these

272 two strains $[\Delta y f h S+y f h S+Y p s A(+$ inducer): $5.60 \pm 2.47 \mu \mathrm{m}($ Fig. 4R); $\Delta y f h S+y f h S+$

273 YpsA-GFP (+ inducer): $7.25 \pm 3.64 \mu \mathrm{m}$ (Fig. 4T)], confirming that YpsA-mediated

274 filamentation requires $\mathrm{YfhS}$. The precise reason for this requirement is unclear at this

275 time.

276

277 DISCUSSION

278 Although many factors involved in facilitating the cell division process have been

279 discovered in B. subtilis (Errington \& Wu, 2017) and E. coli (Du \& Lutkenhaus, 2017),

280 our understanding is still incomplete even in these model organisms as evidence of yet

281 to be uncovered factors exists (Rodrigues \& Harry, 2012, Bailey et al., 2014). We

282 reported previously that YpsA is such a factor, which appears to play a role in cell

283 division in B. subtilis and S. aureus (Brzozowski et al., 2019a). The precise mechanism

284 by which YpsA functions remains unclear. The structure of YpsA and another SLOG

285 superfamily member DprA, a single-stranded DNA binding protein, is similar. Thus it is

286 possible YpsA also binds DNA, or nucleotides such as NAD or ADP-ribose as

287 speculated previously (Brzozowski et al., 2019a). We undertook this study to shed light

288 on the possible pathways through which YpsA functions. In this report, we describe our 289 observations of YpsA-mediated lethality on solid medium and utilized that phenomenon

290 as a tool to isolate spontaneous suppressors. Using this technique, we have isolated

291 intragenic suppressors and an extragenic suppressor that abolishes YpsA-mediated

292 toxicity. 
294 We have isolated four intragenic suppressors (E55D, P79L, R111P, G132E) using our

295 screen. Given that E55 and P79 residues are highly conserved among YpsA, perhaps

296 not surprisingly, mutations in those residues render YpsA inactive at least with respect

297 to its function in cell division. It appears that in the E55D mutation, even though it

298 retains the negative charge, shortening of the side chain appears to weaken the ability

299 to form hydrogen bonds with neighboring residues. The mutations in highly conserved

300 P79 and weakly conserved G132 residues create several steric clashes, which explains

301 why the function of YpsA in cell division is affected. The mutation in the solvent exposed

302 residue R111 may result in weakened intramolecular and/or intermolecular interactions.

303 Thus, our screen has identified multiple key residues that are essential for the proper

304 function of YpsA in regard to cell division inhibition.

305

306 The extragenic suppressor mutation we isolated introduced a premature stop codon in

307 the $y f h S$ open reading frame. YfhS is a relatively small protein (74 amino acids) of

308 unknown function. $y f h S$ is upregulated during sporulation through the SigE transcription

309 factor (Yamamoto et al., 1999) and possibly by AbbA (Banse et al., 2008), thus it has

310 been classified as a sporulation gene. In our results we note that $\Delta y f h S$ cells appear

311 smaller in both width and length compared to our WT control, suggesting that YfhS may

312 have a role in cell size regulation during vegetative growth. Furthermore, we tested and

313 confirmed that YpsA-mediated cell division inhibition is dependent on the presence of

314 full length YfhS. 
316 Given that YfhS is a protein of unknown function, how YfhS and YpsA are linked

317 remains to be determined. During our course of experiments, we noticed that $\Delta y f h S$

318 cells grew slower than our WT control (Fig. S3B). We have previously shown that

319 YpsA-mediated cell division inhibition is a growth rate-dependent phenomenon

320 (Brzozowski et al., 2019a), thus it is possible that the abolition of cell division inhibition

321 in cells lacking $y$ fhS could be attributed to slow growth. However, at this point we cannot

322 rule out the possibility of a direct mechanistic link between YpsA and YfhS, since YpsA

323 plays a role in cell division and YfhS appears to play a role in cell size regulation.

\section{EXPERIMENTAL PROCEDURES}

\section{Strain construction and general methods}

329 laboratory strain PY79 (Youngman et al., 1984). Table S1 contains all relevant strain

330 and oligonucleotide information. The construction of strains overexpressing yps $A$,

$331 y p s A-g f p, y p s A-f l a g$, and ypsA-gfp-flag have been described previously (Brzozowski et

332 al., 2019a). In order to construct a B. subtilis strain containing an inducible copy of $y$ fhS,

333 yfhS was PCR amplified from PY79 chromosomal DNA using primer pair

334 oRB59/oRB60. The resulting PCR product was digested with Sall and Nhel restriction

335 enzymes and cloned into pDR111 (D. Rudner), also digested with Sall and Nhel, to

336 construct plasmid pRB54. The constructed plasmids were then transformed into

337 competent PY79 cells to introduce genes of interest via double crossover homologous

338 recombination into either the native and non-essential amyE locus or into a second 339 amyE locus (bkdB::Tn917::amyE::cat; Amy Camp). 


\section{Media and culture conditions}

342 Overnight $B$. subtilis cultures were grown at $22{ }^{\circ} \mathrm{C}$ in Luria-Bertani (LB) growth medium,

343 and subsequently diluted $1: 10$ into fresh LB medium. Cultures were grown at $37^{\circ} \mathrm{C}$ in a

344 shaking incubator to mid-logarithmic growth phase $\left(\mathrm{OD}_{600}=0.5\right)$, unless otherwise

345 stated. In order to induce the expression of genes under the control of an IPTG-

346 inducible promoter, $250 \mu \mathrm{M}$ IPTG was added to growing cultures, where required, at

347 mid-logarithmic phase, unless stated otherwise.

349 Spot assay

350 All spot assays were completed on LB agar plates supplemented with 1mM IPTG,

351 where required, to induce the expression of genes under the control of an IPTG-

352 inducible promoter. Required strains were first grown to mid-logarithmic phase

$353\left(\mathrm{OD}_{600}=0.5\right)$ at $37^{\circ} \mathrm{C}$ while shaking, and subsequently standardized to an $\mathrm{OD}_{600}=0.1$.

354 Following standardization, serial dilutions of each of the strains were spotted onto the

355 appropriate LB plates at a volume of $1 \mu$ l. Plates were incubated overnight

356 (approximately 14 hours) at $37^{\circ} \mathrm{C}$. On the following day, plates were observed for

357 growth defects.

359 Isolation of spontaneous suppressor mutations

360 The severe growth defect associated with the strain overproducing YpsA-GFP (GG83)

361 allowed for the isolation of spontaneous suppressor mutations that were able to restore

362 growth similar to the WT control. Suppressor mutations were isolated and determined to 
363 be either intragenic or extragenic as indicated in Fig. S2. For this purpose, the strain

364 GG83 was plated on LB agar plates containing $1 \mathrm{mM}$ IPTG to induce the expression of

$365 y p s A-g f p$, and plates were incubated overnight at $37^{\circ} \mathrm{C}$. After overnight incubation,

366 plates were examined for growth defects associated with the overproduction of YpsA-

367 GFP. PY79 was utilized as a control to ensure that any reduction in growth was

368 specifically due to the overproduction of YpsA-GFP. Single colonies of GG83 that did

369 arise in the presence of inducer (likely containing suppressor mutations) were isolated

370 from the original plate and used to inoculate new LB agar plates that were then grown

371 overnight at $37^{\circ} \mathrm{C}$. Genomic DNA was then isolated from each of the strains containing

372 suppressor mutations using standard phenol-chloroform DNA extractions. Isolated

373 genomic DNA was then used to transform WT PY79 cells, which were then screened for

374 integration of $y p s A-g f p$ into the non-essential amyE locus. The resulting transformants

375 were then inoculated onto LB agar plates supplemented with $1 \mathrm{mM}$ IPTG to induce the

376 expression of $y p s A-g f p$, and plates were incubated overnight at $37^{\circ} \mathrm{C}$. On the following

377 day plates were screened for growth defects associated with ypsA-gfp overexpression.

378 PY79 was used as a control to ensure that any observed growth defect was specifically

379 due to the production of YpsA-GFP. If strains harboring an inducible copy of $y p s A-g f p$

380 isolated during our suppressor screen were now able to grow in the presence of the

381 IPTG inducer, then the suppressor mutations were noted as possibly intragenic (yps $A^{*}$ -

$382 \mathrm{gfp})$. If the strains were still unable to grow in the presence of the IPTG inducer, then the

383 mutations were labeled as possibly extragenic - as this indicated that the inducible copy

384 of $y p s A-g f p$ within the amyE locus did not contain any mutations that were able to

385 restore WT-like growth. All strains determined to contain intragenic suppressor 
mutations within the IPTG-inducible copy of $y p s A-g f p$ at the amyE locus were screened via fluorescence microscopy to ensure GFP fluorescence, ruling out some potential mutations within the promoter region, frame-shift mutations, and introduction of premature stop codons. Genomic DNA was isolated from each of the $y p s A^{*}$-gfp strains containing intragenic suppressor mutations and was then used as a template for PCR

391 using primer pair oP106/oP24 to amplify the ypsA-gfp within the amyE locus. The

392 resulting PCR products were sequenced using a 3' internal GFP sequencing primer

393 (oP212) by GENEWIZ (South Plainfield, NJ). Sequence analysis was completed using 394 ApE Plasmid Editor (v2.0.51) (M. Wayne Davis), and multiple sequence alignments were built using Clustal Omega multiple sequence alignment software (Sievers et al.,

396 2011). All strains characterized as containing extragenic suppressor mutations that

397 restored WT-like growth to strains overproducing YpsA-GFP were subjected to

398 additional screening prior to whole genome sequencing by integrating a new copy of

$399 y p s A$-gfp into the amyE locus. The original copy of $y p s A$-gfp was first replaced by a

400 chloramphenicol resistance cassette, and the resulting strain was then transformed with 401 pGG28 (Brzozowski et al., 2019a), to reintroduce a new copy of ypsA-gfp into the amyE

402 locus. The resulting strains were then used to inoculate LB agar plates supplemented

403 with $1 \mathrm{mM}$ IPTG to verify that they were still able to grow in the presence of inducer

404 unlike the GG83 parental strain. PY79 and GG83 were used as controls on these

405 plates. Plates were incubated overnight at $37^{\circ} \mathrm{C}$ and observed for any growth defects

406 associated with YpsA-GFP overproduction on the following day. Genomic DNA was

407 isolated from strains containing extragenic suppressor mutations using the Wizard 
408 Genomic DNA Purification Kit (Promega) and sent for whole genome sequencing (Tufts

409 University School of Medicine Genomics Core).

\section{Bioinformatics and variant detection}

412 Data was analyzed using CLC Genomics Workbench 11 (Qiagen Bioinformatics). First,

413 raw reads were aligned to the PY79 reference sequence (CP00681) using the Map

414 Reads to Reference tool. Output read mappings were then subject to coverage analysis

415 and variant detection. The Basic Variant Detection tool was used to generate a variant

416 track and variant table output in consideration with coverage results. Resultant amino

417 acid changes of variants unique to extragenic suppressors were examined using the

418 Amino Acid Changes tool (version 2.4) using set for genetic code parameter 11:

419 Bacterial, Archaeal, and Plant Plasmid. Subsequently, suppressor mutations were also 420 verified manually.

421

\section{Structural analysis}

423 All figures and rotamers were generated using PyMOL (Schrödinger, LLC).

\section{Growth Curves}

426 PY79, RB314, and RB409 were first grown to mid-logarithmic phase $\left(\mathrm{OD}_{600}=0.5\right)$ in LB

427 broth at $37^{\circ} \mathrm{C}$ with shaking and subsequently standardized to an OD $600=0.1$. IPTG was

428 added to the growth medium at a final concentration of $1 \mathrm{mM}$, where required to induce

429 the expression of genes of interest. Cultures were then grown in LB medium at $37^{\circ} \mathrm{C}$ 
430 with shaking for a total elapsed time of 6 h. Growth curves were plotted using GraphPad

431 Prism version 8.3.1 (GraphPad Software, La Jolla, California, USA).

432

\section{Microscopy}

434 Microscopy was completed by taking $1 \mathrm{ml}$ aliquots of $B$. subtilis cultures and washing

435 with $1 \mathrm{X}$ phosphate buffered saline (PBS) through centrifugation. Cells were then

436 resuspended in $100 \mu$ l of PBS, and the red membrane stain FM4-64 was added at a

437 final concentration of $1 \mu \mathrm{g} / \mathrm{ml}$. The sample was prepared for microscopy by spotting $5 \mu \mathrm{l}$

438 of the cell suspension onto the glass coverslip of a MatTek glass bottom dish and

439 subsequently covering it with a 1\% agarose pad made with sterile water as described

440 previously (Brzozowski et al., 2019b). All imaging was completed at room temperature

441 inside of an environmental chamber using a GE Applied Precision DeltaVision Elite

442 deconvolution fluorescence microscope. Photos were taken using a Photometrics

443 CoolSnap HQ2 camera. All images were acquired by taking 17 Z-stacks at $200 \mathrm{~nm}$

444 intervals. Images were deconvolved though the SoftWorx imaging software provided by

445 the microscope manufacturer.

447 Immunoblot Analysis

448 B. subtilis strains were grown overnight at $22^{\circ} \mathrm{C}$ in LB growth medium, and then diluted

$4491: 10$ into fresh LB the following day. Cultures were grown to an $\mathrm{OD}_{600}=0.5$ and

450 subsequently induced with $1 \mathrm{mM}$ IPTG where required to induce the expression of the

451 genes of interest. Cultures were then grown to $\mathrm{OD}_{600}=1.0$ and following the induction

452 period, $1 \mathrm{ml}$ aliquots of cultures were centrifuged, and cell lysis was completed by 
453 resuspending the cell pellet in a protoplast buffer containing $0.5 \mathrm{M}$ sucrose, $20 \mathrm{mM}$

$454 \mathrm{MgCl}_{2}, 10 \mathrm{mM} \mathrm{KH}_{2} \mathrm{PO}_{4}$, and $0.1 \mathrm{mg} / \mathrm{ml}$ lysozyme. Samples were incubated at $37^{\circ} \mathrm{C}$ for

$45530 \mathrm{~min}$ and then prepared for SDS-PAGE. Following electrophoresis, samples were

456 transferred onto nitrocellulose membrane and subsequently probed with antibodies

457 against GFP, FLAG (Proteintech Group Inc.), or B. subtilis SigA (M. Fujita), which was

458 used as an internal loading control.

459

460

461

462

463

464

465

466

\section{7}

468

469

470

471

472

473

474

475 Figure 1. Overproduction of YpsA or YpsA-GFP results in a lethal phenotype. (A) Cell 476 morphology of wild type (PY79; WT) cells and cells harboring an IPTG-inducible copy of

477 either ypsA (GG82) or ypsA-gfp (GG83) grown in the absence of inducer or in the 478 presence of $250 \mu \mathrm{M}$ IPTG. Fluorescence of red membrane stain (FM4-64; red) and GFP 
479 (green) are shown. Scale bar: $1 \mu \mathrm{m}$. (B) Spot assays of WT cells (PY79) and cells

480 containing an inducible copy of either ypsA (GG82) or ypsA-gfp (GG83). Serially diluted

481 standardized cultures were spotted on plates containing no inducer (left panel) or $1 \mathrm{mM}$

482 IPTG (right panel) and grown overnight at $37^{\circ} \mathrm{C}$. Corresponding dilution factors are

483 indicated below.

484

485 Figure 2. Isolation of spontaneous suppressors. (A-J) Fluorescence microscopy

486 comparing cells containing an inducible copy ypsA-gfp (GG83) and cells containing

487 intragenic mutations (yps $A^{*}$-gfp) isolated during the suppressor screen that resulted in

488 single amino acid changes: G132E (RB300), P79L (RB301), R111P (RB328), and E55D

489 (RB327). Cells were grown in the absence $(\mathbf{A}, \mathbf{C}, \mathbf{E}, \mathbf{G}, \mathbf{I})$ or in the presence $(\mathbf{B}, \mathbf{D}, \mathbf{F}, \mathbf{H}$,

$490 \mathrm{~J})$ of $250 \mu \mathrm{M}$ IPTG. Fluorescence of FM4-64 (red) and GFP (green) are shown. Scale

491 bar: $1 \mu \mathrm{m}$. (K) Spot assays of strains harboring an IPTG inducible copy of ypsA-gfp

492 (GG83) or ypsA*-gfp (RB300, RB301, RB328, RB327) grown without inducer (top

493 panel) or with $1 \mathrm{mM}$ IPTG (bottom panel). Corresponding dilution factors are shown on

494 top. (L) Stability of YpsA-GFP and YpsA*-GFP variants were confirmed when cells were

495 grown in the presence of inducer. Cell lysates were probed via immunoblotting using

496 anti-GFP and anti-SigA (loading control) antisera.

497

498 Figure 3. Structural analysis of the intragenic suppressors. (A) Crystal structure of $B$.

499 subtilis YpsA (PDB ID: 2NX2) with sites of mutation colored in orange. (B-E)

500 Computationally generated mutants are shown in yellow. Hydrogen bonds are shown as

501 black dashes, while steric clashes are represented as red dashes. For clarity, only the 
502 most severe clashes are indicated with interatomic distances less than $2.2 \AA$. (B) The

503 P79L mutation generates severe clashes with multiple surrounding residues. (C) The

504 E55D mutation in the putative DNA-binding groove results in the potential loss of five

505 hydrogen bonds, destabilizing this region. (D) The G132E mutation, similar to P79L

506 mutant, involves a core residue that cannot accommodate any large side chains without

507 severe steric clashes. (E) The R111P mutant eliminates a salt bridge with E114 and

508 produces a clash with the adjacent $\mathrm{H} 110$ backbone. As a surface residue, it also

509 potentially disrupts intermolecular interactions and signaling.

511 Figure 4. Deletion of $y$ fhS rescues YpsA-mediated toxicity and associated filamentation.

512 (A-B) Spot assay of WT cells (PY79), $\Delta y f h S$ cells (RB314), $\Delta y f h S+y f h S(R B 409)$ cells,

513 and cells overexpressing either ypsA or ypsA-gfp in an otherwise wild type background

514 (GG82 and GG83), a $\Delta y f h S$ background (RB288 and RB289), or in a $\Delta y f h S$

515 complementation strain where an intact copy of $y$ fhS is engineered to be under the 516 control of an IPTG-inducible promoter at an ectopic locus (RB410 and RB411). Cultures

517 were standardized and serial dilutions were spotted on solid medium without inducer (A)

518 or with $1 \mathrm{mM}$ IPTG (B). Corresponding dilution factors are indicated on top. (C-T)

519 Fluorescence microscopy comparing cell morphologies of WT cells (PY79), $\Delta y f h S$ cells

520 (RB314), $\Delta y f h S+y f h S$ (RB409) cells, and cells overexpressing either ypsA or ypsA-gfp

521 in an otherwise wild type background (GG82 and GG83), a $\Delta y f h S$ background (RB288

522 and RB289), or in a $\Delta y f h S$ complementation strain (RB410 and RB411). Cells were

523 imaged in the absence of inducer (C, E, G, I, K, M, O, Q, S) or in the presence of 250

$524 \mu \mathrm{M}$ IPTG (D, F, H, J, L, N, P, R, T). Fluorescence signal of FM4-64 membrane dye (red) 
525 and GFP (green) are shown. Scale bar: $1 \mu \mathrm{m}$. (U) Cell lengths of strains shown in

526 panels $\mathbf{C}-\mathbf{T}$ were quantified. The corresponding mean value and standard deviations

$527(n=100)$ are shown.

528

529

530

531

532

533

534

535

536

537

538

539

540

541

542

Table S1. Strains and oligonucleotides used in this study.

Figure S1. Analysis of YpsA accumulation. (A) Fluorescence microscopy of strains containing an IPTG-inducible copy of either ypsA-flag (RB121) or ypsA-gfp-flag (RB125) grown in the absence of inducer (left panels) or in the presence of $250 \mu \mathrm{M}$ IPTG (right panels). Fluorescence of FM4-64 membrane dye (red) and GFP (green) are shown. Scale bar: $1 \mu \mathrm{m}$. (B) Spot assays including wild-type cells (PY79) and cells containing an IPTG-inducible copy of either ypsA (GG82), ypsA-gfp (GG83), ypsA-flag (RB121), or ypsA-gfp-flag (RB125). Dilutions of standardized cultures were spotted on solid medium without inducer (left panel) or containing 1 mM IPTG (right panel). Corresponding dilution factors are indicated below. (C) Anti-FLAG and anti-SigA (loading control) immunoblots of RB121 (YpsA-FLAG), RB412 ( yfhS + YpsA-FLAG), RB125 (YpsAGFP-FLAG), and RB413 ( $\Delta y f h S+$ YpsA-GFP-FLAG) cell lysates. The FLAG/SigA ratios corresponding to each lane are shown.

543

544 Figure S2. Flow chart detailing the methodology used to screen spontaneous 545 suppressor mutations.

546

547 Figure S3. Analysis of $y f h S$ extragenic suppressor mutation. (A) Pairwise alignment of 548 the $y$ fhS sequence in WT (PY79) and the extragenic suppressor (RBSS6E11). The 
549

550

551

552

553

554

555

556

557

558

559

560

561

562

563

564

565

566

567

568

569

570

571

572

573

source of 10-nucleotide duplication is highlighted. (B) Growth curves of WT (PY79), $\Delta y f h S$ (RB314), and $y f h S$ complementation strain (RB409) are shown.

\section{REFERENCES}

1. Bailey, M.W., Bisicchia, P., Warren, B.T., Sherratt, D.J., and Mannik, J. (2014) Evidence for divisome localization mechanisms independent of the Min system and SImA in Escherichia coli. PLoS Genet 10: e1004504.

2. Baker, C.M., and Grant, G.H. (2007) Role of aromatic amino acids in proteinnucleic acid recognition. Biopolymers 85: 456-470.

3. Banse, A.V., Chastanet, A., Rahn-Lee, L., Hobbs, E.C., and Losick, R. (2008) Parallel pathways of repression and antirepression governing the transition to stationary phase in Bacillus subtilis. Proc Natl Acad Sci U S A 105: 15547-15552.

4. Baruah, A., and Biswas, P. (2014) The role of site-directed point mutations in protein misfolding. Phys Chem Chem Phys 16: 13964-13973.

5. Brzozowski, R.S., Huber, M., Burroughs, A.M., Graham, G., Walker, M., Alva, S.S., Aravind, L., and Eswara, P.J. (2019a) Deciphering the Role of a SLOG Superfamily Protein YpsA in Gram-Positive Bacteria. Front Microbiol 10: 623.

6. Brzozowski, R.S., White, M.L., and Eswara, P.J. (2019b) Live-Cell Fluorescence Microscopy to Investigate Subcellular Protein Localization and Cell Morphology Changes in Bacteria. $J$ Vis Exp.

7. Burroughs, A.M., Zhang, D., Schaffer, D.E., lyer, L.M., and Aravind, L. (2015) Comparative genomic analyses reveal a vast, novel network of nucleotide-centric 
systems in biological conflicts, immunity and signaling. Nucleic Acids Res 43:

575 10633-10654.

8. Du, S., and Lutkenhaus, J. (2017) Assembly and activation of the Escherichia coli divisome. Mol Microbiol 105: 177-187.

9. Errington, J., and Wu, L.J. (2017) Cell Cycle Machinery in Bacillus subtilis. Subcell Biochem 84: 67-101.

10. Eswara, P.J., and Ramamurthi, K.S. (2017) Bacterial Cell Division: Nonmodels Poised to Take the Spotlight. Annu Rev Microbiol 71: 393-411. insights into the dynamic bacterial Z ring. Nat Rev Microbiol 14: 305-319.

12. Ramagopal, U.A., Alvarado, J., Dickey, M., Reyes, C., Toro, R., Bain, K., Gheyi, T., Sauder, J.M., Burley, S.K., and Almo, S.C. (2006) Crystal structure of hypothetical protein YpsA from Bacillus subtilis. To be published. are not required for identifying the division site in Bacillus subtilis but ensure its efficient utilization. PLoS Genet 8: e1002561.

14. Sievers, F., Wilm, A., Dineen, D., Gibson, T.J., Karplus, K., Li, W., Lopez, R., McWilliam, H., Remmert, M., Soding, J., Thompson, J.D., and Higgins, D.G. (2011) Fast, scalable generation of high-quality protein multiple sequence alignments using Clustal Omega. Mol Syst Biol 7: 539. into the unique single-stranded DNA-binding mode of Helicobacter pylori DprA. Nucleic Acids Res 42: 3478-3491. 
16. Yadav, T., Carrasco, B., Serrano, E., and Alonso, J.C. (2014) Roles of Bacillus subtilis DprA and SsbA in RecA-mediated genetic recombination. J Biol Chem 289: $27640-27652$.

600

17. Yamamoto, H., Mori, M., and Sekiguchi, J. (1999) Transcription of genes near

601 the sspE locus of the Bacillus subtilis genome. Microbiology 145 ( Pt 8): 2171-

602 2180.

603

18. Youngman, P., Perkins, J.B., and Losick, R. (1984) Construction of a cloning site near one end of Tn917 into which foreign DNA may be inserted without affecting transposition in Bacillus subtilis or expression of the transposon-borne erm gene. Plasmid 12: 1-9.

19.Zhu, B., and Stulke, J. (2018) SubtiWiki in 2018: from genes and proteins to functional network annotation of the model organism Bacillus subtilis. Nucleic Acids Res 46: D743-D748. 
A bioRxiv preprint doi: $\mathrm{https}: / /$ doi.org/10.1101/2020.02.12.946632; this version posted February 13,2020 . The copyright holder for this preprint - Induce (which was not certified by peer review) is the autpqrffudnderefll rights reserved. No reuse allowed without permission.
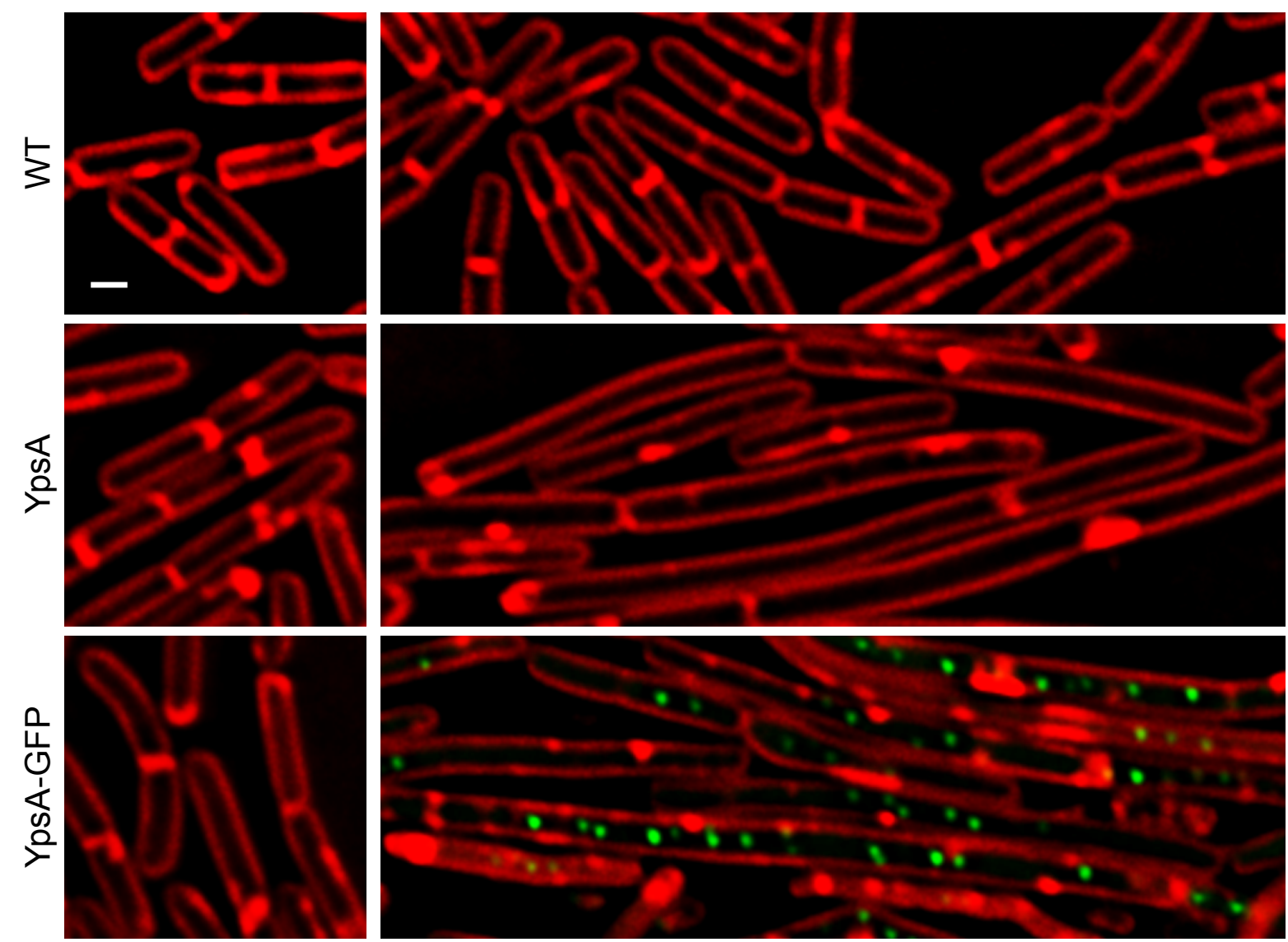

B

- Inducer

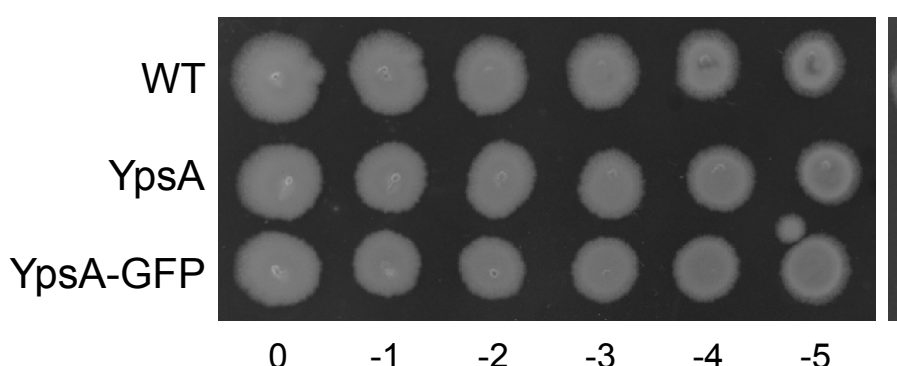

+ Inducer

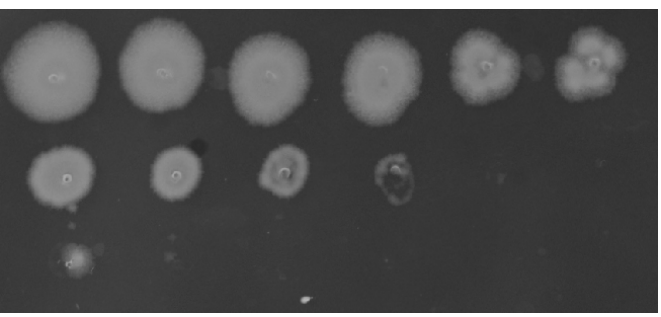

$-5$ 
- In

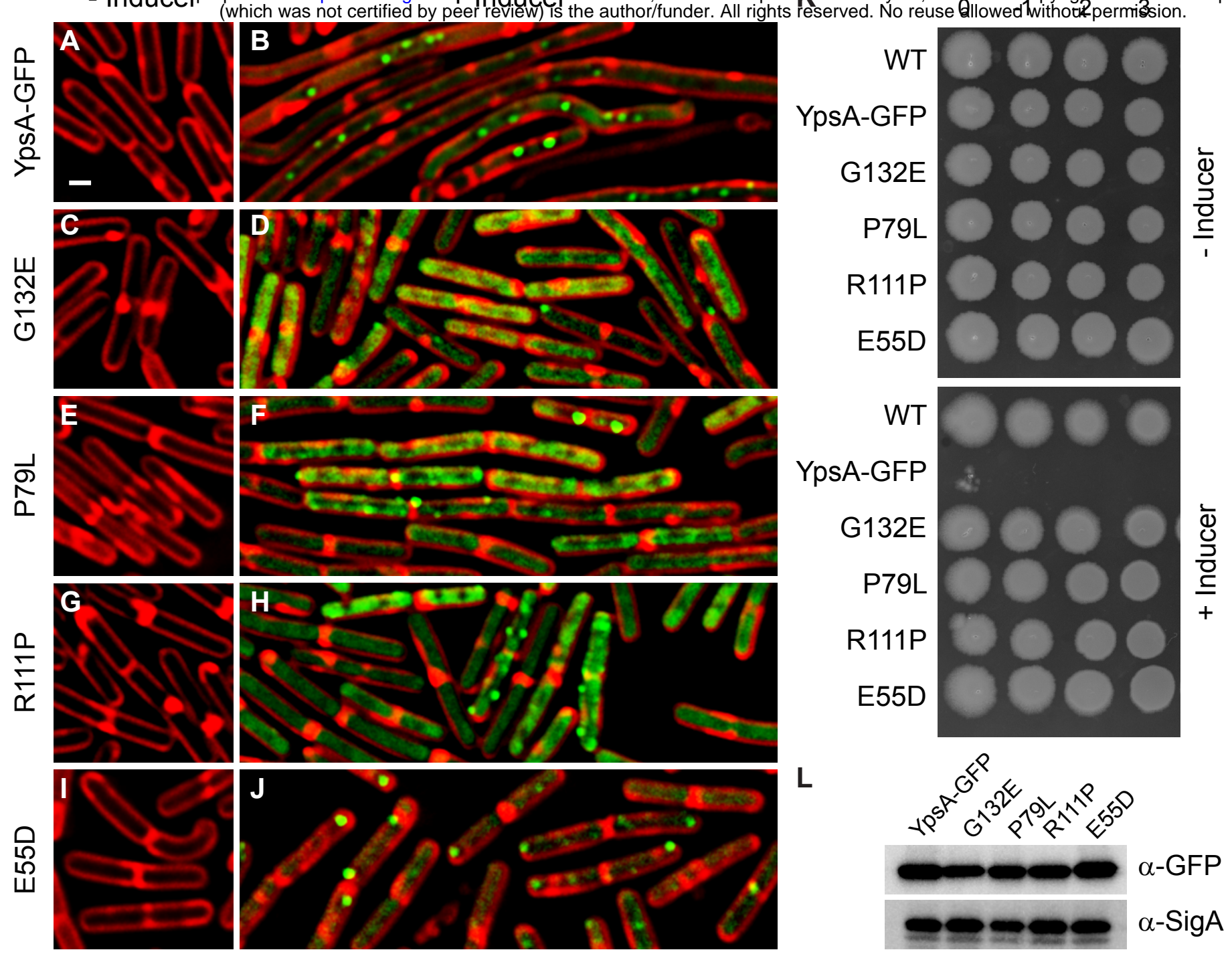


bioRxiv preprint doi: https://doi.org/10.1101/2020.02.12.946632; this version posted February 13, 2020. The copyright holder for this preprint (which was not certified by peer review) is the author/funder. All rights reserved. No reuse allowed without permission.

A

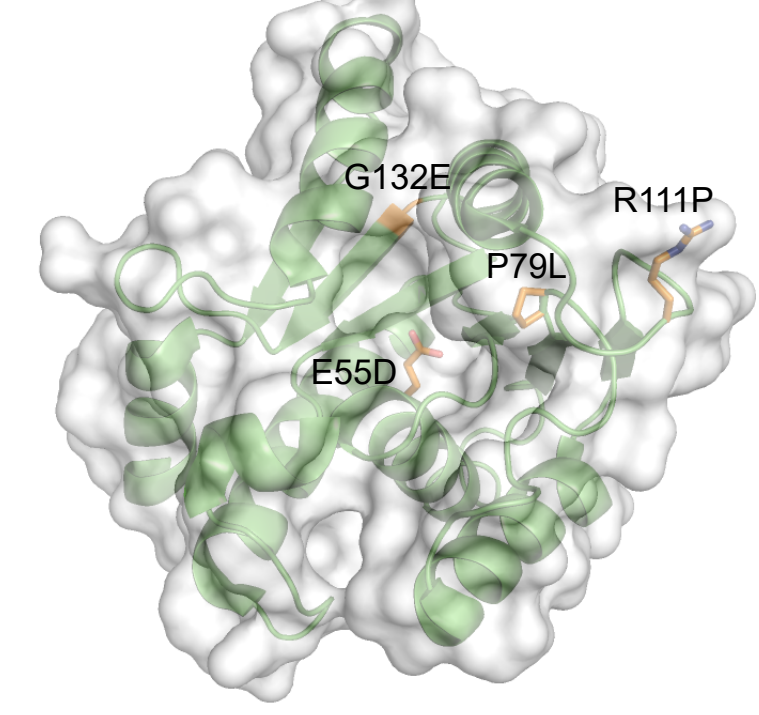

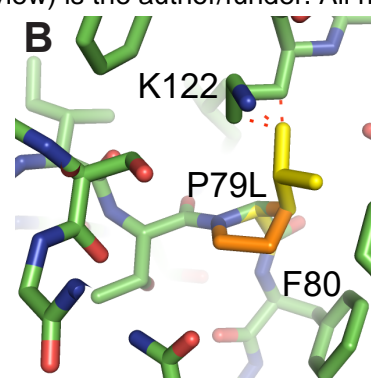

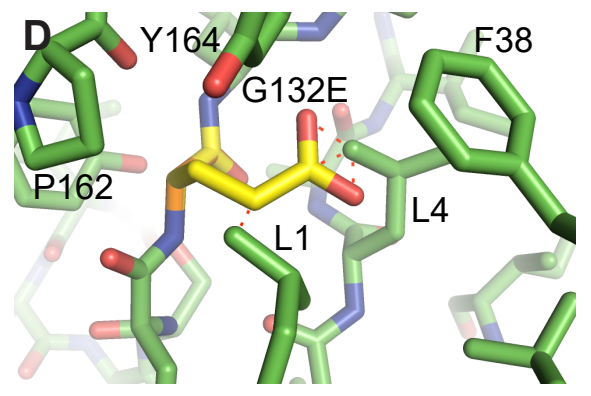

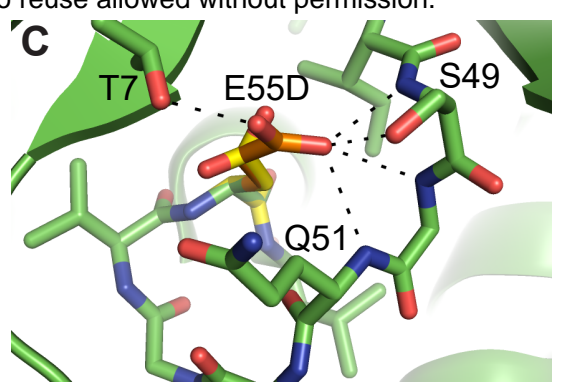

E

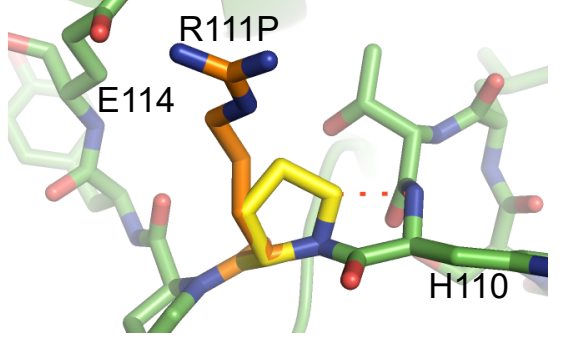



(which was not çrtifiedlby pęr retßew) is the author/funer. All rights reserved. No reuse allowed without pertnission. -3
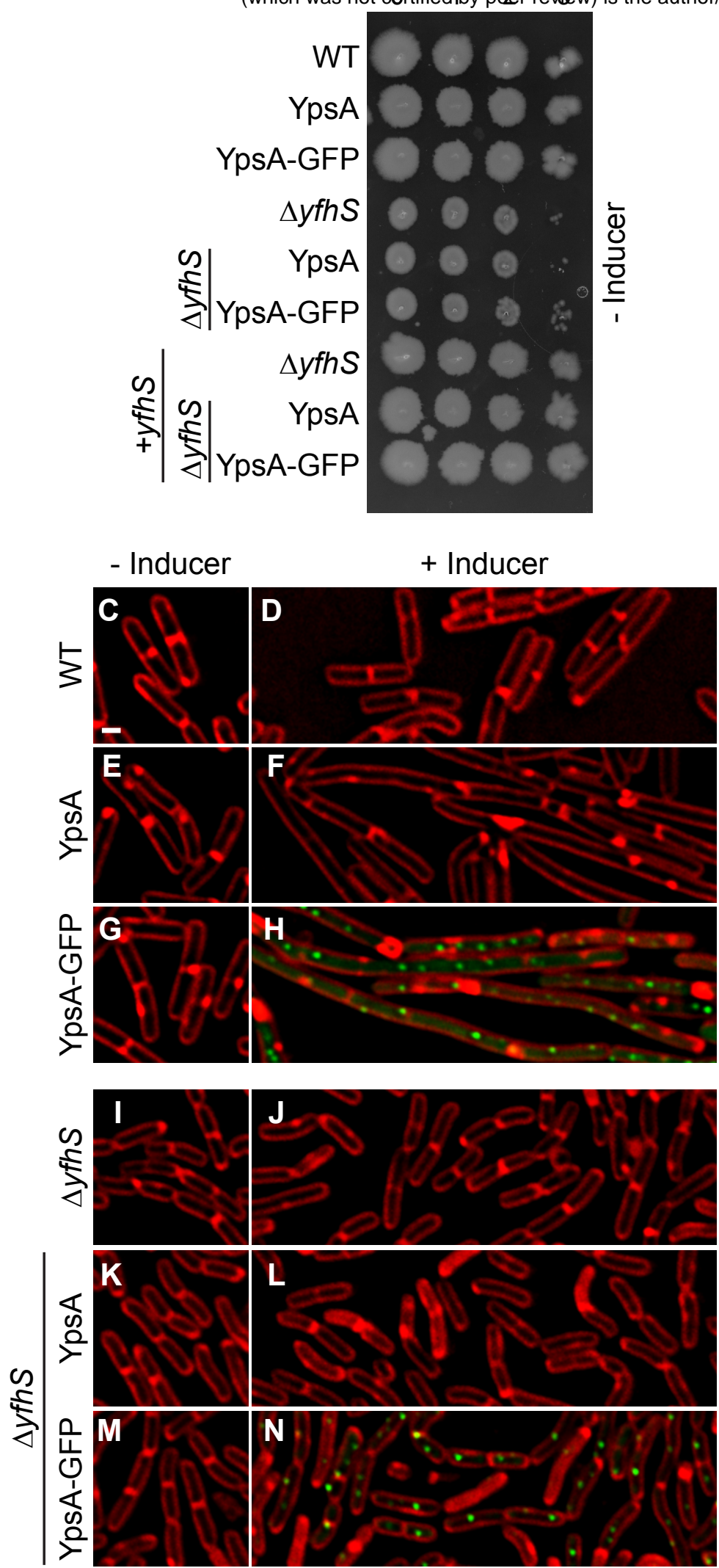
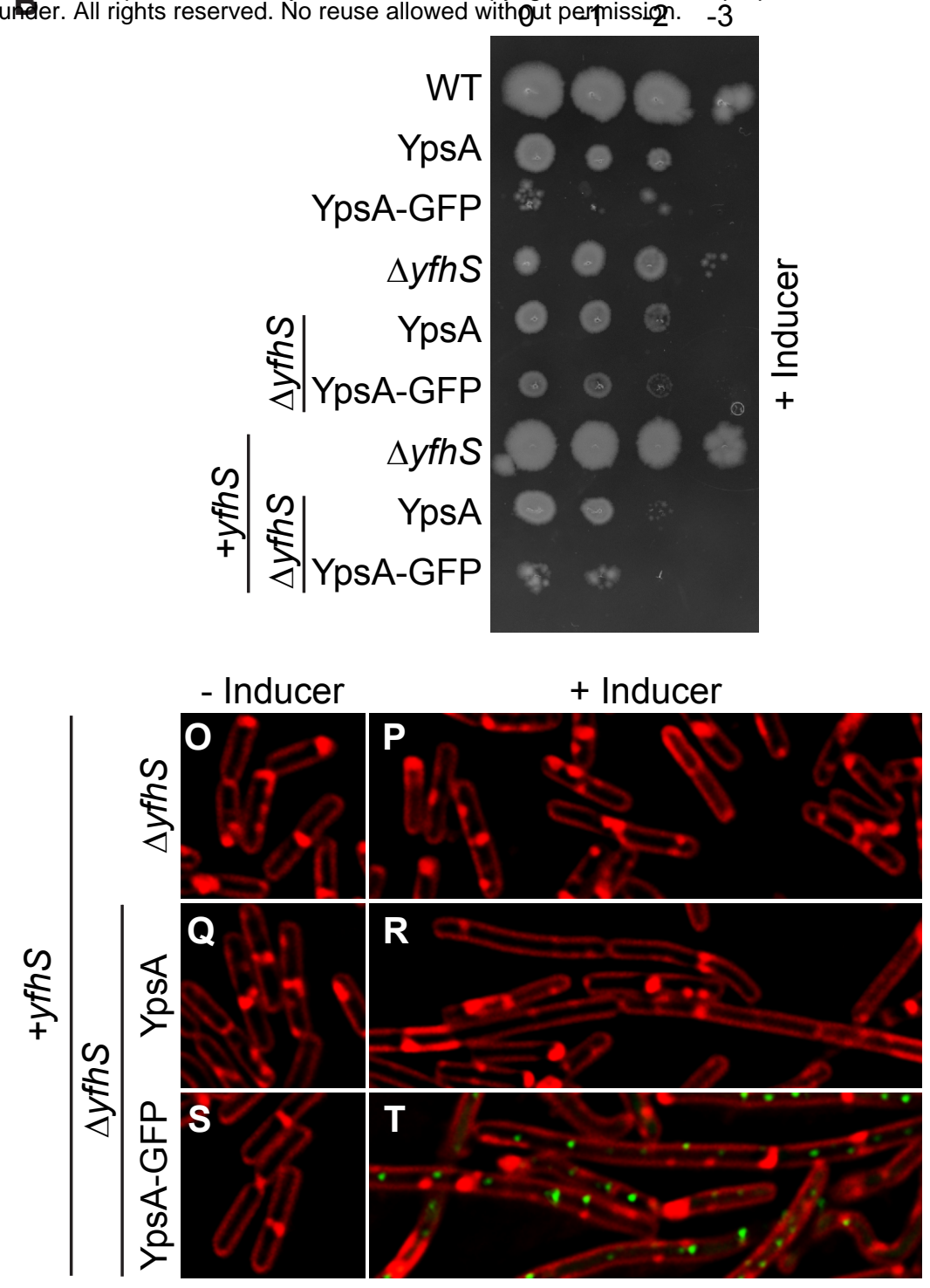

$\mathbf{U}$

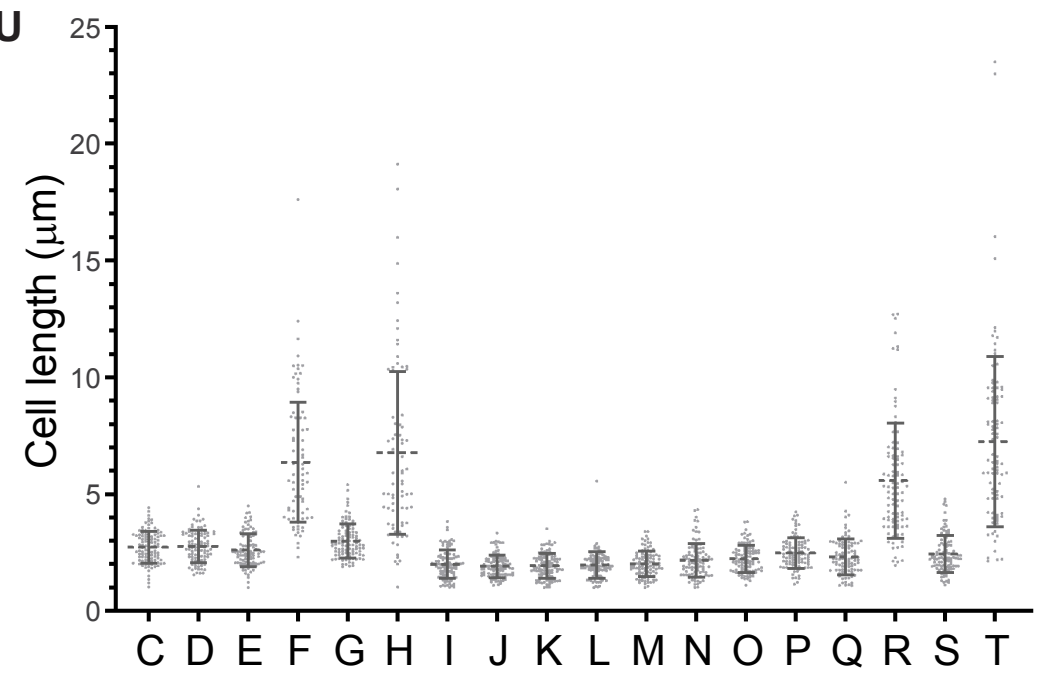

